\title{
Load-Similar Node Distribution for Prolonging Network Lifetime in PMRC-based Wireless Sensor Networks
}

\author{
Qiaoqin $\mathrm{Li}^{1,2}$, Mei Yang ${ }^{1}$, Yan Jin ${ }^{1}$, Jun Zheng ${ }^{3}$, Yingtao Jiang ${ }^{1}$, Jiazhi Zeng ${ }^{2}$ \\ ${ }^{1}$ Dept. of Electrical and Computer Engineering, Univ. of Nevada, Las Vegas, NV, 89154, \\ USA \\ ${ }^{2}$ College of Computer Science and Technology, Univ. of Electronic Science and Technolo- \\ gy, Chengdu, 610054, P. R. China \\ ${ }^{3}$ Dept. of Computer Science and Engineering, New Mexico Institute of Mining and Tech- \\ nology, Socorro, NM 87801, USA \\ Emails: ${ }^{1}$ \{meiyang@egr.unlv.edu, jinyanhit@gmail.com, yingtao@egr.unlv.edu\}, \\ ${ }^{2}$ \{helenli803@uestc.edu.cn, jzzeng@uestc.edu.cn\}, ${ }^{3}$ zheng@cs.nmt.edu
}

\begin{abstract}
In this paper, the energy hole problem in Progressive Multi-hop Rotational Clustered (PMRC)-based wireless sensor networks (WSNs) is studied. We first analyze the traffic load distribution in PMRC-based WSNs. Based on the analysis, we propose a novel load-similar node distribution strategy combined with the Minimum Overlapping Layers (MOL) scheme to solve the energy hole problem in PMRC-based WSNs. Simulation results demonstrate that the load-similar node distribution strategy significantly prolongs network lifetime than an existing nonuniform node distribution and uniform node distribution strategies. The analysis model and the proposed load-similar node distribution strategy have the potential to be applied to other multi-hop WSN structures.
\end{abstract}

Keywords: Wireless sensor networks, energy hole problem, load-similar node distribution

\section{Introduction}

Due to the benefits of low cost, rapid deployment, self-organization capability and cooperative data processing, wireless sensor networks (WSNs) have been proposed as a practical solution for a wide range of applications [1], such as military surveillance and habitat monitoring, etc. A typical WSN is formed by a large number of sensor nodes responsible for sensing data and a sink node responsible for collecting and processing data. Since the energy supply for each sensor node is usually extremely limited, energy efficiency is the primary challenge of WSNs. Previous research works show that clustered structure [2] and multi-hop routing [3] achieve better energy efficiency for large scale WSNs.

In WSNs, the data traffic follows a many-to-one communication pattern. Nodes closer to the sink tend to carry heavier traffic load, which will deplete their energy 
faster. Authors in [4] argue that by the time nodes closest to the sink deplete their energy, nodes farther away to the sink may still hold up to $93 \%$ of their initial energy assuming all nodes have the same fixed transmission range and nodes are uniformly distributed in the network. In the literature, this problem is referred as the energy hole problem and a number of research related to this problem have been conducted.

Olariu and Stojmenović investigate the theoretical aspects of uneven energy depletion problem in sink-based WSNs [5]. Li and Mohapatra develop a mathematical model to analyze the energy hole problem in many-to-one networks [6]. Perillo et al. propose a general model to study the optimal transmission range distribution to maximize the network lifetime [7]. In [8], a nonuniform node distribution strategy is proposed to avoid the energy hole problem in multi-layered sensor networks with fixed layer boundary.

In our previous work [9], a highly scalable network architecture, named as Progressive Multi-hop Rotational Clustered (PMRC) structure, is proposed for the construction of large scale WSNs. In PMRC-based network, nodes are partitioned into layers according to their distance to the sink. A cluster is composed of sensor nodes in the same layer and a cluster head in the upstream layer. Fig. 1 shows the structure of a PMRC-based network with 3 layers. The operation of a PMRC-based network is divided into rounds. Each round begins with a network formation phase when clusters are formed, followed by a data gathering phase.

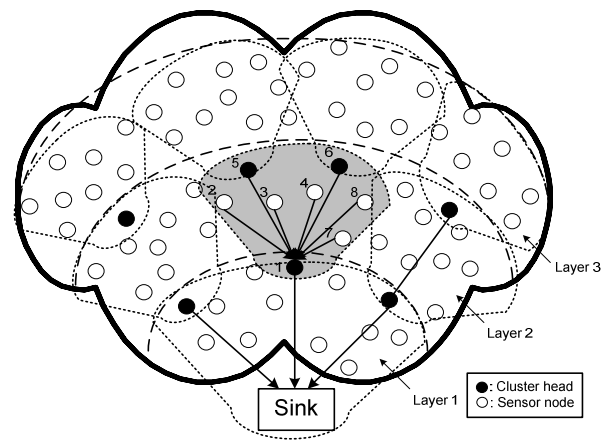

Fig. 1. Structure of a PMRC-based WSN.

Like other multi-hop structures, PMRC also suffers from the energy hole problem. To attack this problem, the Overlapping Layers (OL) scheme is proposed to balance the relay load at the cluster heads for all layers by overlapping the neighbor layers following a desired overlap range [10]. However, due to the fixed layer boundary and overlap range in the OL scheme, the network lifetime is still limited by some nodes with only one candidate cluster head. To overcome this limit, the Minimum Overlapping Layers (MOL) scheme [11] is proposed to gradually increase the required minimum overlap between neighbor layers during network lifetime. As the traffic follows a many-to-one pattern, the network lifetime of MOL-enabled PMRCbased WSNs is still limited by the number of sensor nodes in the initial first layer.

As addressed in [8], node distribution strategy is rather important in balancing energy consumption in multi-hop static sensor networks with single sink node. In this paper, we propose a load-similar node distribution strategy to solve the energy hole 
problem for the PMRC-based WSNs. First, the load analysis in the continuous space of the network is performed, which reflects the gradual change of the layer boundary in the MOL scheme. Then based on the analysis, the load-similar node distribution strategy is proposed. Simulation results confirm the superiority of the proposed loadsimilar node distribution over the nonuniform node distribution [8] and uniform node distribution strategies for MOL-enabled PMRC-based WSNs.

The rest of this paper is organized as follows. In Section 2, the traffic load in PMRC-based WSNs is analyzed. The load-similar node distribution strategy is described in Section 3. Simulation results are presented in Section 4 and the paper is concluded in Section 5.

\section{Analysis of Load}

\subsection{Energy Model}

In our model, each sensor node is assumed to have the same initial energy, whereas the energy of the sink is much higher. Assume that any sensor node may be elected as a cluster head. The energy consumed (referred as load in later text) by each sensor node majorly consists of three parts:

- $E_{t}$ : the energy consumed for transmitting data generated from all sensor nodes in its cluster and the data relayed through all outer layers;

- $E_{r}$ : the energy consumed for receiving data collected from all outer layers;

- $E_{c}$ : the energy consumed for network formation in each round.

Following the free space channel model used in [2], the energy consumed for transmitting $l$-bit data over the distance of $r$ is given by

$$
l\left(E_{\text {elec }}+\varepsilon r^{2}\right),
$$

and the corresponding energy consumed in receiving $l$-bit data is $l E_{\text {elec }}$. According to [2], the system parameters used in in this paper are set as, $E_{\text {elec }}=50 \mathrm{~nJ} / \mathrm{bit}$, $\varepsilon=10 \mathrm{pJ} / \mathrm{bit} / \mathrm{m}^{2}$.

The energy consumed in network formation phase is majorly composed of the energy consumed for receiving control packets, including Control Packets (CP), Header Selection Packets (HP), Broadcast Packet (BP), and Cluster Control Packets (CCP) [9]. Here the energy consumed in sending these packets is neglected due to the small volume of such packets. The energy consumed during each network formation at layer $i, E_{c i}$, can be calculated as:

$$
\begin{gathered}
E_{c 1}=\left(2 * c s+h s^{*} h n\right) E_{\text {elec }}, \\
E_{c i}=(c s * c n+b s * b n+h s * h n+c c s * c c n) E_{\text {elec }}, \quad i>1,
\end{gathered}
$$

where $c s, h s, b s$ and $c c s$ represent the packet length of a CP, HP, BP and CCP, respectively, while $c n, h n, b n$ and $c c n$ represent the respective average number of CPs, HPs, BPs and CCPs received by each node during each network formation. 


\subsection{Analysis of Load}

Assume static sensor nodes are uniformly distributed with node density $\rho$ within a $2 R \times 2 R$ square area, and the sink is located at the center of the area. Assume all sensor nodes are active in transmission and a portion of these nodes (referred as source nodes in the later text) are active in sensing data. Each source node generates and sends $\lambda$ bits of data per unit time. The ratio of the number of source nodes to the total number of sensor nodes is $\mu$.
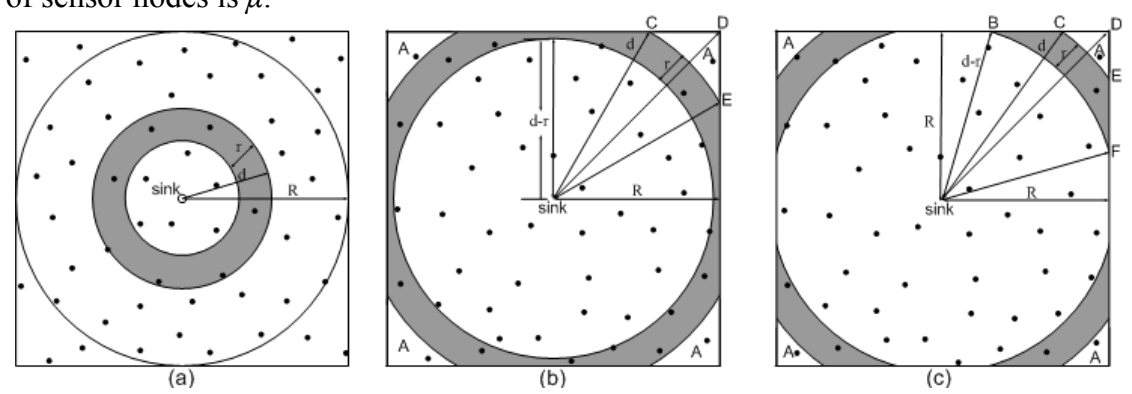

Fig. 2. Geometry relationship of layer $i, d \leq R$ (a), $R<d \leq R+r$ (b), $R+r<d \leq \sqrt{2} R$ (c).

Fig. 2 illustrates the geometry relationship of layer $i$ (with radius $r$ which is equal to the transmission range) within the $2 R \times 2 R$ square area. Assume the distance from the sink to the outer boundary of layer $i$ is $d$. First, we deduce the average load per node in layers within the range of $r<d \leq R$, as shown in Fig. 2(a). According to the energy model, the total energy consumed for data receiving and transmission in a unit time by all the sensor nodes in layer $i(i>1), E_{r i}$ and $E_{t i}$, are given by:

$$
\begin{gathered}
E_{r i}=E_{\text {elec }} \lambda \rho \mu\left(4 R^{2}-\pi d^{2}\right), \quad i>1, \quad r<d \leq R, \\
E_{t i}=\left(E_{\text {elec }}+\varepsilon r^{2}\right) \lambda \rho \mu\left(4 R^{2}-\pi(d-r)^{2}\right), \quad i>1, \quad r<d \leq R .
\end{gathered}
$$

Assume $N_{i}$ is the number of sensor nodes in layer $i$, which can be calculated by $\rho \pi\left(d^{2}-(d-r)^{2}\right)$. Then the average load per node in layer $i$ (for $i>1$ and $r<d \leq R$ ) in a unit time is given by:

$$
\begin{aligned}
L_{i}= & \frac{E_{r i}+E_{t i}}{N_{i}}+\frac{E_{c i}}{T_{r}}=\frac{E_{\text {elec }} \lambda \rho \mu\left(4 R^{2}-\pi d^{2}\right)}{\rho \pi\left(d^{2}-(d-r)^{2}\right)}+\frac{\left(E_{\text {elec }}+\varepsilon r^{2}\right) \lambda \rho \mu\left(4 R^{2}-\pi(d-r)^{2}\right)}{\rho \pi\left(d^{2}-(d-r)^{2}\right)}+ \\
& \frac{\left(c s * c n+b s * b n+h s^{*} h n+c c s * c c n\right) E_{\text {elec }}}{T_{r}},
\end{aligned}
$$

where $T_{r}$ is the average lifetime per round, and $\frac{1}{T_{r}}$ gives the number of network formations performed in a unit time.

For $R<d \leq R+r$, shown as the shaded area in Fig. 2(b), the average load per node in layer $i$ in a unit time can be calculated as:

$$
L_{i}=\frac{E_{r i}+E_{t i}}{N_{i}}+\frac{E_{c i}}{T_{r}}
$$




$$
=\frac{4 \lambda \mu E_{\text {elec }} A}{S_{1}}+\frac{\lambda \mu\left(E_{\text {elec }}+\varepsilon r^{2}\right)\left(4 A+S_{1}\right)}{S_{1}}+\frac{\left(c s * c n+b s^{*} b n+h s^{*} h n+c c s * c c n\right) E_{\text {elec }}}{T_{r}},
$$

where $A$ gives the area of each corner outside the shaded area, which can be calculated by $\left(R-\sqrt{d^{2}-R^{2}}\right) R-\left(\frac{\pi}{4}-a \cos \left(\frac{R}{d}\right)\right) d^{2}$, and $S_{1}$ is the area of the shaded layer which can be calculated by $4\left(R^{2}-\frac{\pi}{4}(d-r)^{2}-A\right)$.

For $R+r<d<\sqrt{2} R$, shown as the shaded area in Fig. 2(c), the average load per node in layer $i$ in a unit time can be calculated as:

$$
\begin{aligned}
L_{i} & =\frac{E_{r i}+E_{t i}}{N_{i}}+\frac{E_{c i}}{T_{r}} \\
& =\frac{\lambda \mu E_{\text {elec }} A}{A^{\prime}-A}+\frac{\lambda \mu\left(E_{\text {elec }}+\varepsilon r^{2}\right) A^{\prime}}{A^{\prime}-A}+\frac{\left(c s^{*} c n+b s^{*} b n+h s^{*} h n+c c s * c c n\right) E_{\text {elec }}}{T_{r}},
\end{aligned}
$$

where $A^{\prime}$ gives the area of shape $B C D E F$, which can be calculated as $\left(R-\sqrt{(d-r)^{2}-R^{2}}\right) R-\left(\frac{\pi}{4}-a \cos \left(\frac{R}{d-r}\right)\right)(d-r)^{2}$, and the calculation of the area of each corner $A$ follows that in (6-2).

For $d=\sqrt{2} R$, i.e., at the outermost layer, the average load per node in layer $i$ in a unit time can be calculated as:

$$
L_{i}=\frac{E_{t i}}{N_{i}}+\frac{E_{c i}}{T_{r}}=\mu \lambda\left(E_{\text {elec }}+\varepsilon r^{2}\right)+\frac{(c s * c n+b s * b n+c c s * c c n) E_{\text {elec }}}{T_{r}} .
$$

For sensor nodes at layer 1, they will receive and forward the data coming from outside layer 1 and also send the data generated from layer 1. Then we have:

$$
\begin{aligned}
& E_{r 1}=E_{\text {elec }} \lambda \rho \mu\left(4 R^{2}-\pi r^{2}\right), \\
& E_{t 1}=\left(E_{\text {elec }}+\varepsilon r^{2}\right) \lambda \rho \mu 4 R^{2} .
\end{aligned}
$$

Here, $L_{1}$ is obtained as:

$$
L_{1}=\frac{E_{r 1}+E_{t 1}}{N_{1}}+\frac{E_{c 1}}{T_{r}}=\frac{E_{\text {elec }} \mu \lambda\left(8 R^{2}-\pi r^{2}\right)}{\pi r^{2}}+\frac{\varepsilon \mu \lambda 4 R^{2}}{\pi}+\frac{E_{\text {elec }}\left(2 * c s+h s^{*} h n\right)}{T_{r}} .
$$

Fig. 3 depicts the average load vs. $d$ normalized in units of $r=40 m$ with $\mu=20 \%$ and $\lambda=1600 \mathrm{bps}$. The values of cs (145bits), hs (169bits), bs (205bits), and ccs (259bits) are set same as in the simulations. Other parameters, $h n=3.4, b n=6.79$, ccn $=3.4$, and $T_{r}=25 \mathrm{~s}$, are estimated based on the analysis of the simulation result with uniformly distributed sensor nodes for $r=40 \mathrm{~m}$ and $\rho=0.0064$, and these values need to be adjusted for different $r$ and $\rho$ values. As shown in the figure, the average load per sensor node shows a sharp decrease with respect to the increase of the distance between the sensor node and the sink.

Consistent with our intuition, sensor nodes at layer 1 experience the heaviest load as they have to forward all the data traffic outside layer 1. When the sensor nodes close to the sink node deplete their energy, a ring-like "hole" surrounding the sink node is created, and the sensor nodes outside the "hole" area are actually separated 
from the sink. As such, the network lifetime is upper-bounded by the total energy of the sensor nodes within layer 1 for the MOL-enabled PMRC-based WSN.

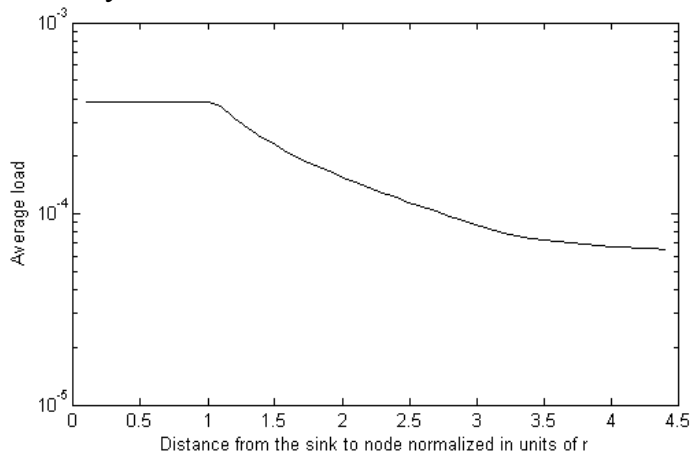

Fig. 3. Load distribution in MOL-enabled PMRC-based WSNs.

\section{Load-Similar Node Distribution}

Based on the above analysis, to solve the energy hole problem in the MOL-enabled PMRC-based WSN, we propose to use a novel load-similar node distribution strategy. The underlying principle is that if the sensor nodes are deployed in the area according to the load distribution (that is, more nodes will be deployed in the range where the average load is higher), then the load among different layers in the sensor network tends to be balanced.

During the deployment of the network, the location of a node will be determined as follows. The distance from this node to the sink is randomly generated and a random load value is also generated. If the distance is less than or equal to the transmission range (i.e., the node is within the first layer), the node is deployed. Otherwise, if the generated load value is less or equal to the load calculated based on the distance, the node is deployed, or discarded otherwise. This process repeats until all the sensor nodes in the network are deployed.

Compared with the node distribution strategy proposed in [8], the node deployment in our strategy is very straightforward. The network formation and routing is simply based on the PMRC structure with the MOL scheme. Hence, there is no need to deploy the forwarding nodes deliberately. In addition, using the cluster structure, each node simply sends its data to its cluster head. This is a sharp contrast to the $q$-Switch routing [8], where each node needs to select one relay node with the most residual energy out of up to $q$ possible forwarding nodes each time before it actually sends out its data. This puts extra requirement that the forwarding nodes periodically broadcast their residual energy. 


\section{Performance Evaluation}

To evaluate the performance of the proposed load-similar node distribution strategy for the MOL-enabled PMRC-based WSN, extensive simulations have been conducted on the WSN simulation module developed on OPNET modeler [12]. In all simulations, we assume a $250 \mathrm{~m}$ × $250 \mathrm{~m}$ geographical area covered by a sensor network with the sink node located at the center. Table 1 lists the key parameters used in the simulations.

Table 1. Simulation parameters

\begin{tabular}{|l|l|}
\hline Parameter & Value \\
\hline Number of nodes & 400 \\
\hline Radio transmission range & $\{40,60,80,100\} \mathrm{m}$ \\
\hline Initial energy per node & $0.5 \mathrm{~J}$ \\
\hline Packet generation rate & $1 \mathrm{pkt} / \mathrm{s}$ \\
\hline Packet length & 200 Bytes \\
\hline Simulation time & Until network partition \\
\hline
\end{tabular}

The following performance metrics are collected.

Time to first node death: in our simulation, we consider only the node death due to drained energy. Generally, this metric reflects the worst lifetime.

Network lifetime: it is defined as the time when the network is no longer connected or all source nodes drain out their energy.

Number of network formations: it is defined as the number of network formations.

Average residual energy: it is defined as the average residual energy of all sensor nodes in their initial layer when the network lifetime ends.

In the following, we present the simulation results of the aforementioned performance metrics for three different node distribution strategies: 1) load-similar node distribution, where sensor nodes are deployed following the load distribution analysis in Section 2; 2) nonuniform node distribution [8], where the number of nodes distributed in adjacent coronas $C_{i}$ and $C_{i+1}$ is initially regulated as $N_{i} / N_{i+1}=q$ with a common ratio of $q=2$; and 3 ) uniform node distribution, where sensor nodes are uniformly distributed in the area. The location of each node in these three distributions is generated using Matlab. In each simulation, a portion of sensor nodes ( $20 \%$ in our simulations) are selected as source nodes to generate and send data. Without loss of generality, these source nodes are randomly distributed in the square area. The results shown are the averaged results of 5 sets of source nodes.

Fig. 4 shows the time to first node death vs. transmission range $r$. In general, the time to first node death for all distributions shows an increasing trend with the increase of $r$. The first node death typically happens in the first layer. With the increase of $r$, the load carried by the nodes in layer 1 decreases because they will consume less energy in receiving packets as there are less nodes distributed outside layer 1 though they will consume more energy in transmitting packets. Some abrupt changes are attributed to the fact that the initial network topology also has a significant impact to the time to first node death.

Fig. 5 shows the network lifetime for all distributions increases monotically with the increase of $r$. With the increase of $r$, the number of candidate cluster heads for each layer increases, which helps prolonging the network lifetime. Under the same transmission range, load-similar distribution achieves longer network lifetime than 
uniform distribution (by up to 32\%) and nonuniform distribution (by up to $73 \%$ ) when $r<80 \mathrm{~m}$. This confirms that the load-similar distribution is more suitable for PMRCbased network than the other two distributions. When $r \geq 80 \mathrm{~m}$, there is no significant difference between the network lifetime for the three distributions as the network lifetime ends when all source nodes are exhausted.

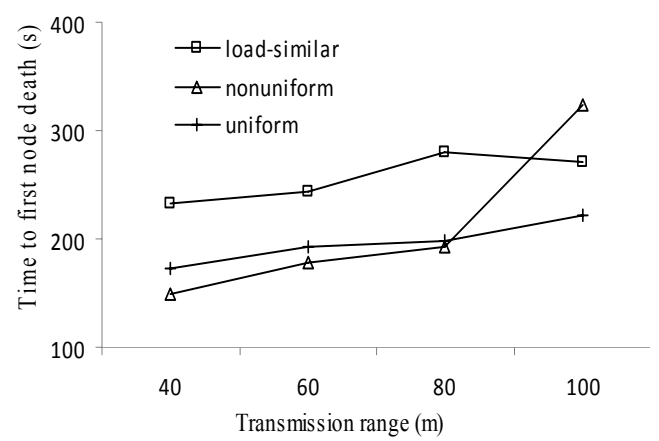

Fig. 4. Time to first node death vs. transmission range.

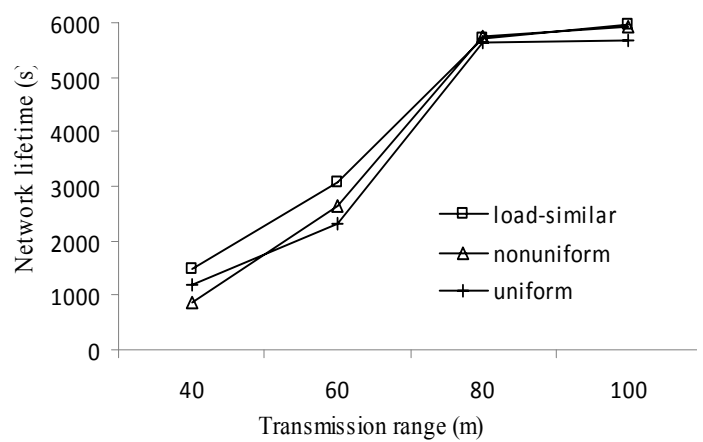

Fig. 5. Network lifetime vs. transmission range.

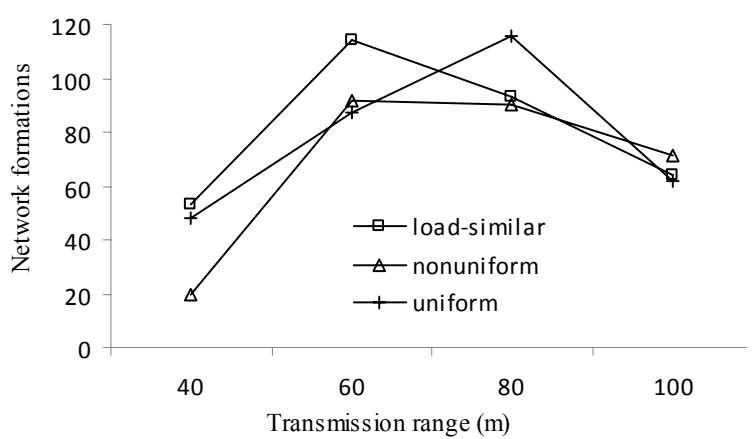

Fig. 6. Number of network formations vs. transmission range. 


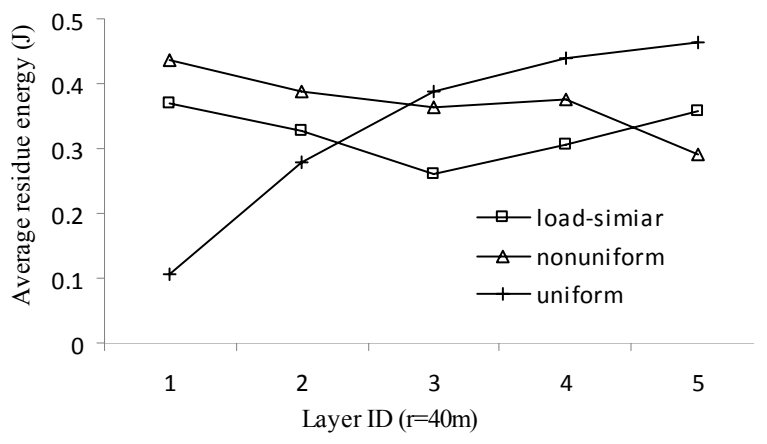

Fig. 7. Average residue energy at $r=40 \mathrm{~m}$.

Fig. 6 presents the number of network formations vs. transmission range $r$. Generally, the number of network formations shows an increasing trend followed by a decreasing trend with the increase of $r$. When the transmission range is getting larger, more candidate cluster heads are available, which leads to more number of network formations. For $r \geq 80 \mathrm{~m}$, the average load at layer 1 is decreased, which leads to the longer average time per round (i.e., the average time between two network formations). As a result, the number of network formations drops.

Fig. 7 shows the average residual energy vs. layer ID when $r=40 m$. Layers with larger layer ID are the ones far from the sink. The residual energy at each node is directly related to the load carried by each node. The average residual energy of uniform distribution shows an increasing trend with the increase of layer ID, which is consistent with the load distribution analysis. The residual energy of both the loadsimilar distribution and nonuniform distribution is better balanced than uniform distribution in most layers. However, the lifetime of nonuniform distribution is shorter than that of load-similar distribution and eventually more energy is wasted.

\section{Conclusion}

In this paper, the energy hole problem in PMRC-based WSNs is studied. We first analyze the traffic load distribution in PMRC-based WSNs and show that the average load per sensor node increases with the decrease of distance from the sink. Based on the analysis, we propose a novel load-similar node distribution strategy combined with the MOL scheme to alleviate the energy hole problem in PMRC-based WSNs. Extensive simulations have been conducted to validate the analysis. The simulation results confirm that the proposed load-similar node distribution strategy achieves good energy balance among different layers in the network and prolongs network lifetime than an existing nonuniform node distribution and uniform node distribution strategies. The superiority of the load-similar node distribution strategy is more evident when there are more number of layers in the network. 


\section{Acknowledgements}

We would like to thank OPNET Tech. Inc. for providing license of OPNET Modeler with wireless module.

\section{References}

1. I. F. Akyildiz, W. Su, Y. Sankarasubramaniam, E. Cayirci.: A survey on sensor networks. IEEE Commu. Mag. 147, 102-114 (2002)

2. W. Heinzalmen, A. Chandrakasan, H. Balakrishman.: An application-specific protocol architecture for wireless microsensor networks. IEEE Trans. Wireless Communications. 1 (4), 660-670 (2002)

3. J. Ding, K. Sivalingam, R. Kashyaoa, L. J. Chuan.: A multi-layered architecture and protocols for largescale wireless sensor networks. In: $58^{\text {th }}$ SVTC, pp. 1443-1447. Piscataway, N. J. (2003)

4. A. Wadaa, S. Olariu, L. Wilson, M. Eltoweissy, K. Jones.: Training a wireless sensor network. Mobile Networks and Applications. 51-168 (2005)

5. S. Olariu, I. Stojmenovic.: Design guidelines for maximizing lifetime and avoid energy holes in sensor networks with uniform distribution and uniform reporting. In: $25^{\text {th }}$ INFOCOM, pp. 1-12. NewYork (2006)

6. J. Li, P. Monhapatra.: Analytical modeling and mitigation techniques for the energy hole problem in sensor networks. Pervasive and Mobile Computing. 3(8), 233-254 (2007)

7. M. Perillo, Z. Cheng, W. Henzelman. On the problem of unbalanced load distribution in wireless sensor networks. In: IEEE GLOBECOM, pp. 74-79. (2004)

8. X. Wu, G. Chen, S. K. Das.: Avoiding energy holes in wireless sensor networks with non-uniform node distribution. IEEE Trans. Parallel and Distributed System. 9(5), 710-720 (2008)

9. M. Yang, S. Wang, A. Abdelal, Y. Jiang, Y. Kim.: An improved multi-layered architecture and its rotational scheme for large-scale wireless sensor networks. In: $4^{\text {th }}$ IEEE CCNC, pp. 855-859. (2007)

10. H. Wang, M. Yang, Y. Jiang, S. Wang, L. Gewali.: Overlapped layers for prolonging network lifetime in multi-hop wireless sensor networks. In: $5^{\text {th }}$ ITNG, pp. 755-760. IEEE Computer Society (2008)

11. Q. Li, M. Yang, H. Wang, Y. Jiang, J. Zeng.: Minimum overlapping layers and its variant for prolonging network lifetime in PMRC-based wireless sensor networks. In: $5^{\text {th }}$ IEEE CCNC, pp. 1-5. (2008)

12. OPNET Modeler, OPNET Tech. Inc., http://www.opnet.com. 